ORIGINAL ARTICLE

\title{
Effects of a home exercise programme on shoulder pain and functional status in construction workers
}

\section{P M Ludewig, J D Borstad}

See end of article for authors' affiliations

......................

Correspondence to: A/Prof. P M Ludewig, Program in Physical Therapy, Department of Physical Medicine \& Rehabilitation, The University of Minnesota MMC 388, 420 Delaware Street, Minneapolis, MN 55455, USA; ludew001@umn.edu

Accepted 14 November 2002
Background: Repetitive or sustained elevated shoulder postures have been identified as a significant risk factor for occupationally related shoulder musculoskeletal disorders. Construction workers exposed to routine overhead work have high rates of shoulder pain that frequently progresses to functional loss and disability. Exercise interventions have potential for slowing this progression.

Aims: To evaluate a therapeutic exercise programme intended to reduce pain and improve shoulder function.

Methods: Construction worker volunteers were screened by history and clinical examination to test for inclusion/exclusion criteria consistent with shoulder pain and impingement syndrome. Sixty seven male symptomatic workers (mean age 49) were randomised into a treatment intervention group $(\mathrm{n}=34$ ) and a control group ( $\mathrm{n}=33$ ); asymptomatic subjects $(\mathrm{n}=25)$ participated as an additional control group. Subjects in the intervention group were instructed in a standardised eight week home exercise programme of five shoulder stretching and strengthening exercises. Subjects in the control groups received no intervention. Subjects returned after 8-12 weeks for follow up testing.

Results: The intervention group showed significantly greater improvements in the Shoulder Rating Questionnaire (SRQ) score and shoulder satisfaction score than the control groups. Average post-test SRQ scores for the exercise group remained below levels for asymptomatic workers. Intervention subjects also reported significantly greater reductions in pain and disability than controls.

Conclusions: Results suggest a home exercise programme can be effective in reducing symptoms and improving function in construction workers with shoulder pain.
$\mathrm{R}$ epetitive or sustained shoulder elevation during occupational tasks has been identified as a significant risk factor for shoulder tendonitis or non-specific shoulder pain. ${ }^{1-3}$ The evidence of occupational risk is strongest for combined exposure to multiple physical factors, such as holding a tool while working overhead. ${ }^{1}$ Construction workers in many trades, by the nature of their work, have substantial exposure to awkward postures, repetitive and forceful muscular contractions, and overhead work. ${ }^{4}$ However, not all workers exposed to these conditions develop shoulder symptoms, with prevalence rates in various construction trades ranging from $25 \%$ to $71 \%{ }^{4}$

Various theories exist regarding additional factors that may interact with occupational exposure and contribute to the development of shoulder pain. ${ }^{5-7}$ Shoulder impingement, defined as compression and irritation of the rotator cuff structures as they pass beneath the coracoacromial arch during elevation of the $a r m,{ }^{68}$ is believed to be a primary mechanism of occupationally related shoulder pain. Repetitive impingement can result in shoulder tendonitis, and if not alleviated, can progress to tears of the rotator cuff tendons. ${ }^{8}$ Regardless of the initial aetiology, altered shoulder motions are believed to exacerbate shoulder tendonitis conditions. $^{6-11}$ Impingement is thought to be due to inadequate subacromial space for clearance of the rotator cuff tendons as the arm is elevated. Therefore, motion abnormalities that further minimise this space are believed detrimental to the condition. These motion abnormalities are thought to be important factors in influencing which persons within occupations with substantial exposure to overhead work will develop impingement syndromes and rotator cuff dysfunction.

Abnormal motions that bring the humeral head and rotator cuff tendons in closer contact with the coracoacromial arch during arm elevation are particularly problematic in impingement syndromes. These motions include decreased scapular upward rotation about an axis approximately perpendicular to the scapular plane, decreased posterior tipping about an axis approximately parallel to the scapular spine, and decreased external rotation about the humeral long axis. ${ }^{10}$ Additionally, increased humeral anterior or superior translations as the arm is elevated are believed to compromise the available subacromial space. ${ }^{11}$ Previous investigation compared construction workers with predominately chronic (greater than three months duration) shoulder symptoms to asymptomatic controls from the same trades. ${ }^{10}{ }^{11}$ Decreased scapular posterior tipping and upward rotation, increased anterior translations of the humerus, and decreased cross body adduction range of motion were found in the symptomatic group. ${ }^{10}{ }^{11}$ These changes are consistent with functional compromise of the subacromial space. Additionally, the alterations in scapular and humeral motion during arm elevation were linked to decreases in muscle activity of the serratus anterior, and increases in activity of the upper and lower trapezius. ${ }^{10}$ Chronic shoulder pain in these workers could result in a tendency to decrease use of the painful shoulder, leading to disuse atrophy and deconditioning, although symptomatic workers report similar percentages of daily overhead work as asymptomatic workers. ${ }^{10}$

Stretching and strengthening programmes are often used in conservative treatment of shoulder pain, attempting to reduce symptoms and alter identified motion and muscle

Abbreviations: ANOVA, analysis of variance; SEM, standard error of the measurement; SRD, smallest real difference; SPADI, Shoulder Pain and Disability Index; SRQ, Shoulder Rating Questionnaire 


\section{Main messages}

- The results of this investigation support the premise that a limited home exercise intervention can be effective in reducing symptoms and improving self reported functional status in construction workers with symptoms of shoulder pain.

- Although significant improvements were obtained, exercise intervention subjects did not obtain similar function and pain scores as the asymptomatic group, and a variation of the intensity, use of alternate exercises, and/or inclusion of modalities may be of greater benefit.

- Further research is needed to determine the most effective exercise programmes to reduce shoulder symptoms and improve function.

activity abnormalities. ${ }^{12}{ }^{13}$ The serratus anterior is a critical muscle to target in an exercise programme, due to its primary role in the control of scapular upward rotation and posterior tipping, as well as the identified reductions in serratus muscle activity in impingement subjects. ${ }^{910}$ On the other hand, excess upper trapezius activity in impingement subjects is believed to contribute to abnormal superior translation and reduced rotation of the scapula. ${ }^{10}$ Therefore, exercise programmes may attempt to reduce upper trapezius activity during humeral elevation. Additionally, the pectoralis minor muscle is frequently stretched, as a tight pectoralis minor would limit normal scapular upward rotation and posterior tipping through its insertion on the coracoid process. ${ }^{10}{ }^{12}$ Posterior capsule stretching is also utilised to allow normal humeral head posterior translation and prevent excess anterior humeral head translation during arm elevation. ${ }^{11}{ }^{12}$ Finally, rotator cuff exercises are generally incorporated in impingement treatment programmes due to the critical functional role of the rotator cuff muscles, particularly the infraspinatus and teres minor. ${ }^{9}$ These rotator cuff muscles stabilise the humeral head on the glenoid, prevent excess superior or anterior humeral head translations, and produce humeral external rotation to clear the greater tuberosity from beneath the acromion. ${ }^{9}$

The purpose of this investigation was to implement and evaluate a therapeutic exercise programme intended to modify the previously identified motion and muscle activity abnormalities believed related to shoulder impingement among construction workers. The specific hypothesis was that construction workers participating in a shoulder home exercise programme would show significant improvements in shoulder function and reductions in shoulder pain compared to a non-intervention control group, but remain significantly below functional levels for an asymptomatic control group from the same population.

\section{METHODS Subjects}

The target population for this study was construction journeymen with regular exposure to overhead work conditions. Construction workers from primarily the sheet metal, electrical, plumbing and pipefitting, and heat and frost insulation trades were included. Painters and carpenters represented a small percentage $(3 \%)$ of the sample. Volunteers were recruited through local unions, and at safety meetings conducted for campus construction employees, representing a convenience sample. Both symptomatic and asymptomatic individuals were invited to participate in the

\section{Policy implications}

- The potential long term value of exercise programmes in reducing rates of rotator cuff surgeries and shoulder medical disability claims in these workers deserves further investigation.

- Employer supervised preventive exercise programmes for reducing occupationally related musculoskeletal symptoms should be further investigated.

study, thus allowing improvements due to intervention effects to be compared to functional levels in asymptomatic workers. Preliminary power analyses based on expected variability within and between groups resulted in minimum sample size estimates of 25 subjects per group to provide $80 \%$ power at an alpha level of 0.05 . The study was approved by the Institutional Review Board at the University of Minnesota, USA, and all subjects signed a written informed consent document prior to participation.

The history of each subject's shoulder symptoms and their demographic data were gathered through a standard set of questions. All subjects were required to have a self reported occupational exposure to overhead work for longer than one year and a minimum of $130^{\circ}$ of active scapular plane abduction as measured goniometrically during a clinical examination. Additional inclusion criteria for symptomatic individuals consisted of a current reported history of shoulder pain localised to the glenohumeral joint region excluding cervical and periscapular pain, but including the common site of referred pain of the rotator cuff to the C5-6 dermatome above the deltoid insertion. For inclusion, symptomatic subjects also had to present with at least two positive shoulder impingement tests (Neer, Hawkins/Kennedy, Yocum, Jobe, and/or Speeds tests $)^{14-16}$ and pain reproduction during two of three additional categories of clinical tests. These categories included: (1) a painful arc on active scapular plane abduction of the $\operatorname{arm}^{17}$; (2) tenderness to palpation of the biceps or rotator cuff tendons; and (3) pain with one or more resisted glenohumeral joint motions (flexion, abduction, internal rotation, or external rotation). Flexion and abduction were resisted at $90^{\circ}$ of elevation, and internal and external rotation were resisted both at the subject's side and at $90^{\circ}$ of abduction.

Symptomatic and asymptomatic subjects were excluded if they: (1) had a history of rotator cuff surgery; (2) reported a history of glenohumeral dislocation, or other traumatic injury to the shoulder; (3) reported only periscapular or cervical pain during arm elevation; or (4) had shoulder symptoms reproduced by a cervical assessment. Eligibility was determined through the clinical examination performed by one of two licensed physical therapists prior to entry into the study. Sensitivity and specificity of individual clinical tests for diagnosing shoulder impingement is variable. ${ }^{15-16}{ }^{18}$ However, when the clinical examination is interpreted as a whole requiring multiple positive tests (impingement tests, painful arc, pain with resisted motion, etc), the accuracy of clinical diagnosis of shoulder impingement compared to arthroscopic confirmation is high. ${ }^{16}$

A total of 110 workers volunteered for the study, and 92 (all male) met the clinical inclusion/exclusion criteria. Symptomatic subjects were randomly assigned to the intervention $(n=34)$ or control $(n=33)$ groups on entry into the study, prior to collection of baseline measurements. Randomisation was performed by an investigator blindly selecting one of two slips of paper indicating group assignment. Asymptomatic subjects $(n=25)$ were all assigned to 
Table 1 Demographic data for each group reported as mean and standard error

\begin{tabular}{|c|c|c|c|}
\hline Variable & Intervention & $\begin{array}{l}\text { Symptomatic } \\
\text { control }\end{array}$ & $\begin{array}{l}\text { Asymptomatic } \\
\text { control }\end{array}$ \\
\hline Age (years) & $4 \varepsilon$ & 49.2 & 49.4 \\
\hline res) & $1.78 i$ & $1.77 i$ & $1.80(0.01)$ \\
\hline Weight (kg) & $87.4(2.2)$ & $89.9(2.7)$ & $90.7(2.1)$ \\
\hline Overhead work (\% of day) & $32.7(3.5)$ & $35.5(4.8)$ & $31.2(4.2)$ \\
\hline Years in trade & $23.7(2.0)$ & $24.3(1.8)$ & $22.7(2.8)$ \\
\hline
\end{tabular}

a second control group. Groups were similar with regard to demographic variables of age, height, weight, years in the trade, and percentage of overhead work (table l). Based on demographic data available from a participating large local union and past investigation of similar unions in another state, ${ }^{4}$ the demographic characteristics of our sample were generally similar to the source population (average age 4149 , average years in the trade $17-24$, gender $98 \%$ male).

\section{Measurements}

All subjects completed the Shoulder Rating Questionnaire (SRQ) (Appendix), and 10 additional questions pertaining to shoulder pain and disability in the occupational setting created using the format of the Shoulder Pain and Disability Index (SPADI) (table 2). Outcome measures were all patient self report, and uniform instructions were given. Psychometric properties (validity, reliability, responsiveness to change) of the SRQ and SPADI have been previously reported for patients with shoulder pathology. ${ }^{19-21}$ High reliability of the modified SPADI occupational pain and disability questions has been previously shown in construction workers (ICC 0.85-0.91) using a test-retest design. ${ }^{22}$ However, the validity and responsiveness of these modified questions has not been previously tested.

\section{Experimental procedure}

All subjects were evaluated for the inclusion/exclusion criteria using a standard clinical examination at the initial visit, and completed the SRQ and work related questions, both at the time of entry into the study (pretest) and at follow up (post-test). At the conclusion of the initial visit, the symptomatic intervention group received instruction in a home exercise programme from one of the licensed therapists. Each subject received written/pictorial instructions for home reference and a daily exercise log to monitor compliance with the exercise programme. Intervention subjects returned one week later to review the exercise programme with the therapist and have questions regarding the exercises addressed. At four weeks, intervention subjects

Table 2 Work related questionnaire items

During the past week, how severe was your pain:

1. At its worst?

2. Working with your hands below shoulder level?

3. Working with your hands at or above shoulder level?

4. Working with your arms fully overhead?

5. Lifting an object or tool weighing 5-10 pounds overhead?

6 . Liffing an object weighing more than 10 pounds overhead?

During the past week, how much difficulty did you have:

7. Working with your hands below shoulder level?

8. Working with your hands at or above shoulder level?

9. Lifting an object weighing 5-10 pounds overhead?

10. Lifting an object weighing more than 10 pounds overhead?

Subjects responded to each question by circling a number on a 1-10 scale. were contacted by telephone to monitor compliance, discuss any problems, and ensure proper progression of the exercises. An optional four week recheck with the therapist was also scheduled if subjects were uncertain about the exercises or having any difficulties performing the exercises. Subjects from all groups returned an average of 10 weeks after the initial visit to complete the SRQ and additional work related questions. The researchers were not blinded to group assignment, but were to baseline measurements at the time of follow up. At the conclusion of the post-test, subjects from the symptomatic control and asymptomatic groups were also instructed in the exercise programme.

Ninety two per cent of subjects completed the study. Seven subjects were lost to follow up, four (11.8\%) in the exercise intervention group, and three control subjects (one symptomatic (3\%) and two asymptomatic (8\%)). One intervention subject withdrew after experiencing a new injury at work that interfered with continuation of the exercises. Another intervention subject was referred by his physician for additional outpatient physical therapy and subsequently withdrew from the study. A third intervention and one symptomatic control subject were not able to return for follow up for personal reasons (death in the family, custody dispute). The remaining three subjects either were no shows or were unable to be reached after multiple attempts at the time of post-test. Subjects lost to follow up were similar to the full sample with regard to demographic characteristics (average age 47, height $1.8 \mathrm{~m}$, weight $89.8 \mathrm{~kg}$, years in the trade 23.0). Sixty one per cent of intervention subjects returned completed exercise $\log$ s at the time of follow up.

\section{Exercise intervention}

Intervention subjects were asked to perform two stretches for 30 seconds each repetition and five repetitions each day (fig $1 \mathrm{~A}-\mathrm{B}$ ). The pectoralis minor stretch was performed by asking the subject to place each hand at shoulder height on adjacent walls of a corner and lean into the corner. The second stretch for the posterior shoulder was performed by reaching towards the opposite scapula and then using the uninvolved hand to further horizontally adduct the humerus until a stretch was achieved. A muscle relaxation exercise for the upper trapezius was performed five times daily by having the subjects raise the arm overhead in the scapular plane without shrugging the shoulder (fig $1 \mathrm{C}$ ). Relaxation was enhanced through visual input by performing the exercise in front of a mirror, or by proprioceptive input by placing the uninvolved hand on the active upper trapezius.

Subjects were instructed to perform progressive resistance strengthening exercises three days per week for two muscle groups. For the serratus anterior muscle, strengthening was performed supine by protracting the scapula and raising a hand held weight superiorly (fig 1D). Humeral external rotation was resisted with Thera-Band (blue resistance level; The Hygenic Corporation, Akron, OH, USA) while subjects were in a standing position (fig $\mathrm{IE}$ ). Subjects were instructed to progress from an initial position of the arm close to their side, to a position of abduction of the arm. For both strengthening exercises, subjects were instructed to perform three sets of 10 repetitions the first week, progress to three sets of 15 repetitions the second week, and three sets of 20 repetitions the third week. After achieving three sets of 20 repetitions for three consecutive sessions, subjects were to further progress their programme by increasing weight resistance or Thera-Band tension (by shortening the band), and repeating the repetition sequence as described. Subjects were instructed that exercises may induce muscle fatigue but should not cause increased shoulder pain. 
A

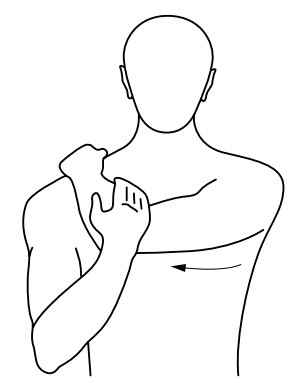

C

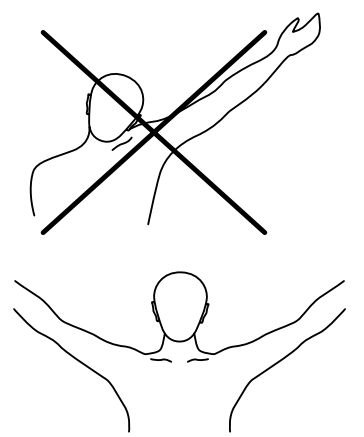

$E$

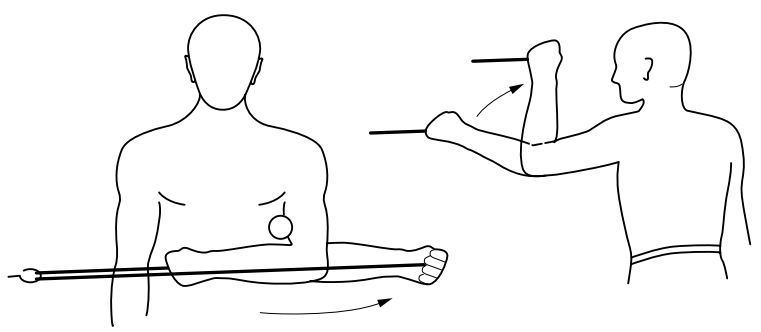

Figure 1 Home exercise programme. A, posterior shoulder stretch; B, pectoralis stretch; $C$, upper trapezius relaxation exercise, avoiding a shoulder shrug while raising the arm; $D$, serratus anterior strengthening exercise; $E$, external rotation strengthening exercise, left is arm at the side; $F$, external rotation strengthening exercise progression with arm abducted.

\section{Data reduction and analysis}

The SRQ was scored using the method described by $L^{\prime}$ Insalata ${ }^{19}$ resulting in scores ranging from 17 to 100, with higher scores indicating greater shoulder function and less shoulder symptoms. The weighting system multiplies the global assessment rating by 1.5 , the pain score by 4 , the daily activity score by 2 , the recreational and athletic activities score by 1.5 , and the work score by $1 .{ }^{19}$ The satisfaction score is a single item, not used to calculate the overall SRQ score, and could range from 1 to 10 , with higher scores indicating greater satisfaction. The responses from the six additional work related pain questions were averaged to create an overall work related pain score ranging from 1 to 10 , with higher scores indicating increased pain with work. The responses from the four additional work related disability questions were also averaged to create an overall work related disability score ranging from 1 to 10 , with higher scores indicating greater difficulty with work performance.

Descriptive data were determined for average changes (post-test - pretest) by group in SRQ, satisfaction, pain and disability scores, as well as average percent changes from pretest scores. For each dependent variable, a two way mixed model analysis of variance (ANOVA) was used to determine the main effects of group (intervention, symptomatic control, or asymptomatic) or time (pretest and post-test), and any interaction effects. The group factor was a between subjects comparison and the time factor a within subjects comparison. A significance level of 0.05 was the criterion for significance. Significant interactions were expected, with anticipated improvements in function and reduction in pain for the intervention group over time, and stable values expected over time for the control groups. In the presence of significant interactions, post hoc analyses with Tukey-Kramer adjustments were completed to test for differences between groups in both pretest and post-test scores. If significant differences between symptomatic groups were present for a dependent variable at pretest, change scores were analysed, comparing between the intervention and control groups after adjusting for the pretest score.

Possible confounding effects were assessed by computing Pearson correlation matrices between demographic data (age, height, weight, years in the trade, and percent overhead work), and the dependent variables. As no demographic variables reached a correlation above 0.48 , none were included as covariates in the analysis.

The initial analysis included all subjects from whom posttest data were obtained, regardless of their level of compliance with the exercise programme. A secondary complete "intention to treat" analysis was also performed where all subjects initially enrolled were analysed. ${ }^{23}$ Missing post-test data were replaced with imputed values based on the average of the observed means from the two symptomatic groups. ${ }^{23}$

\section{RESULTS}

Subjects in the intervention group showed significant improvements from pretest to post-test in their SRQ scores, while control subjects remained essentially stable (table 3), resulting in an interaction of group and time $(\mathrm{F}=9.12$; $\mathrm{p}<0.001$; fig $2 \mathrm{~A}$ ). As post hoc analysis revealed significantly lower pretest SRQ scores in the intervention group, SRQ change scores (table 4) were further analysed for differences between the intervention and symptomatic control groups, adjusting for the initial (pretest) SRQ score. Results of this analysis further showed the significantly improved SRQ scores in the intervention group $(\mathrm{F}=7.66 ; \mathrm{p}<0.01)$. At post-test, all symptomatic subjects' average SRQ scores remained significantly below those of the asymptomatic group (table 3 ).

Satisfaction scores also showed a significant interaction of group and time with significant improvements in satisfaction in the intervention group ( $\mathrm{F}=8.97 ; \mathrm{p}<0.001$; fig $2 \mathrm{~B})$. In this analysis however, there were no pretest differences in scores between the intervention and symptomatic control groups. At post-test, the satisfaction scores of the intervention group were significantly greater than those of the symptomatic control group, but remained significantly less than the asymptomatic group (table 3 ).

Similar patterns of responses across groups were also noted for the work related pain and disability scores with significant interactions of group and time $(\mathrm{F}=13.95, \mathrm{p}<0.001$, fig $2 \mathrm{C}$; and $\mathrm{F}=9.16, \mathrm{p}<0.001$, fig $2 \mathrm{D}$, respectively). Again, in this analysis there were no pretest differences in scores between the intervention and the symptomatic control group. At post-test, the work related pain and disability scores of the intervention group were significantly reduced compared to the symptomatic control group, but remained significantly greater than the asymptomatic group (table 3). Average percent improvements in the intervention group ranged from 19\% for the SRQ score to $44 \%$ for the satisfaction score (table 4 ).

Results of the intention to treat analysis were similar to the primary analysis for most dependent variables, although 
Table 3 Means (standard error of mean) of outcome variables by group

\begin{tabular}{|c|c|c|c|c|c|c|}
\hline \multirow[b]{2}{*}{ Variable } & \multicolumn{2}{|l|}{ Intervention } & \multicolumn{2}{|c|}{ Symptomatic control } & \multicolumn{2}{|c|}{ Asymptomatic control } \\
\hline & Pretest & Post-test & Pretest & Post-test & Pretest & Post-test \\
\hline $\begin{array}{l}\text { SRQ score } \\
\text { Intent to treat }\end{array}$ & $65.9(1.96)$ & $\begin{array}{l}78.0(2.31)^{*} \\
75.8^{*}\end{array}$ & 72.5 (1.99) & $\begin{array}{l}71.1(2.24) \\
71.0\end{array}$ & $93.8(2.28)$ & $94.0(2.64) \ddagger$ \\
\hline $\begin{array}{l}\text { Satisfaction } \\
\text { score }\end{array}$ & $4.5(0.31)$ & $6.2(0.35) \dagger$ & $5.0(0.31)$ & $5.0(0.34)$ & $9.1(0.37)$ & $8.8(0.40) \ddagger$ \\
\hline Intent to treat & & 5.9 & & 4.9 & & \\
\hline $\begin{array}{l}\text { Work related } \\
\text { pain }\end{array}$ & $4.8(0.28)$ & $2.8(0.29) \dagger$ & $4.6(0.28)$ & $4.1(0.29)$ & $1.3(0.32)$ & $1.4(0.34) \ddagger$ \\
\hline Intent to treat & & $3.1 \dagger$ & & 4.2 & & \\
\hline $\begin{array}{l}\text { Work related } \\
\text { disability }\end{array}$ & $4.1(0.30)$ & $2.5(0.29) \dagger$ & $3.8(0.30)$ & $3.7(0.29)$ & $1.3(0.35)$ & $1.3(0.34) \ddagger$ \\
\hline Intent to treat & & $2.8 \dagger$ & & 3.8 & & \\
\hline \multicolumn{7}{|c|}{$\begin{array}{l}\text { Higher scores indicate greater function and increased satisfaction }(S R Q) \text {, and less pain and less disability. } \\
\text { *Indicates significant improvement in score from pretest to post-test }(p<0.01) \text {. } \\
\text { tIndicates significantly different score than symptomatic control group }(p<0.05) \text {. } \\
\text { tIndicates significantly different score than intervention group }(p<0.05) \text {. }\end{array}$} \\
\hline
\end{tabular}

$\mathrm{p}$ values tended to be higher. Interactions of group and time continued to be statistically significant for all variables $(p<0.001)$. SRQ change scores, adjusted for initial SRQ scores, remained significantly higher in the intervention group $(\mathrm{p}<0.001)$. Additional pain and additional disability scores also continued to be significantly lower in the intervention group at post-test compared to the symptomatic control group $(\mathrm{p}<0.05)$. Satisfaction scores for the intervention group at post-test tended to be higher than those of symptomatic control subjects after performing the intention to treat analysis (table 3 ). However, the associated $\mathrm{p}$ value was equal to 0.06 and thus above the a priori critical level for statistical significance.

\section{DISCUSSION}

The results of this investigation support the premise that a limited home exercise intervention can be effective in reducing symptoms and improving self reported functional status in construction workers with symptoms of shoulder pain. The intervention group showed positive changes for all variables, while both control groups remained essentially unchanged over time (table 3). Although exercise programmes are commonly used as conservative treatment for shoulder tendonitis and impingement symptoms, ${ }^{24}$ few randomised controlled studies have assessed the effects of shoulder exercise on symptoms or functional status, ${ }^{12} 25-27$ and to our knowledge, none of these studies have targeted populations with occupationally related shoulder impingement.

The available past clinical trials have shown similar positive effects of exercise for subjects with shoulder pain related to impingement or tendonitis. ${ }^{125-27}$ Bang and Deyle compared exercise in combination with manual therapy to exercise alone, but did not have a non-exercise comparison
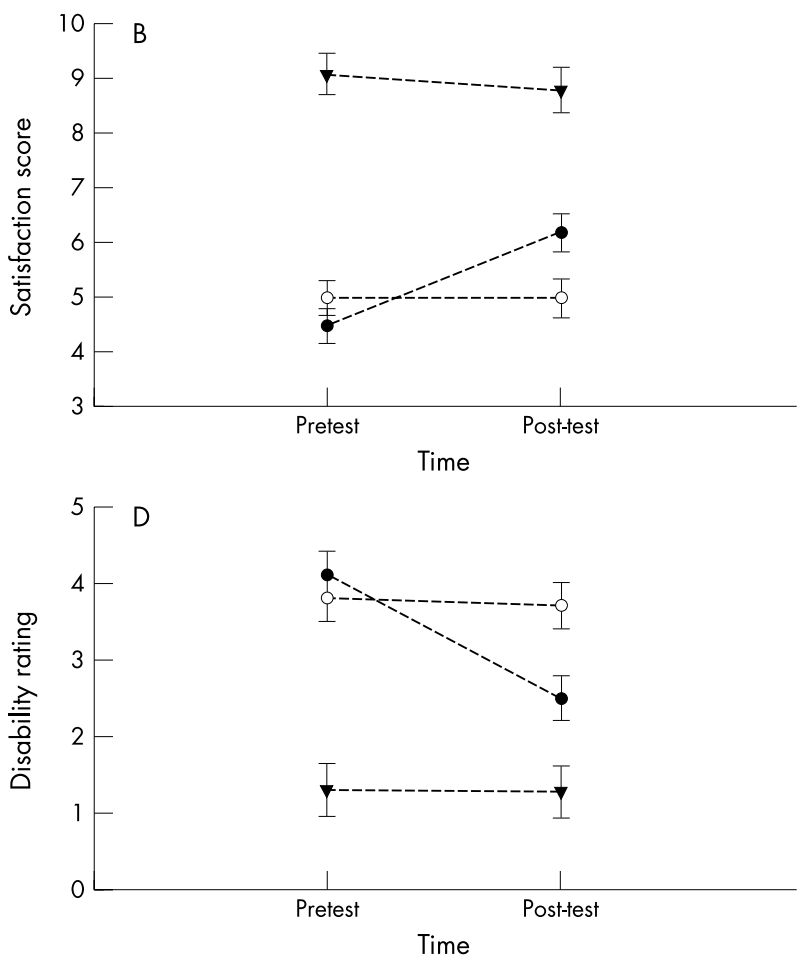

Figure 2 Questionnaire results by group and time. A, Shoulder Rating Questionnaire scores; B, satisfaction score; C, work related pain score; D, work related disability score. Note the changes in scale between graphs and that not all graphs begin with a zero scale. 
Table 4 Change (pretest to post-test) scores and percent change scores in function and pain measures by group

\begin{tabular}{|c|c|c|c|c|c|}
\hline \multirow[b]{2}{*}{ Measurement tool } & \multirow[b]{2}{*}{ Variable } & \multirow[b]{2}{*}{$\begin{array}{l}\text { Range of possible } \\
\text { scores }\end{array}$} & \multicolumn{3}{|l|}{ Group } \\
\hline & & & Intervention & $\begin{array}{l}\text { Symptomatic } \\
\text { control }\end{array}$ & $\begin{array}{l}\text { Asymptomatic } \\
\text { control }\end{array}$ \\
\hline Shoulder Rating Questionnaire & $\begin{array}{l}\text { Difference score mean (SE) } \\
\text { Percent change (SE) }\end{array}$ & $17-100$ & $\begin{array}{l}11.17(2.83)^{*} \\
19.23(4.75)\end{array}$ & $\begin{array}{l}-1.56(2.5) \\
-0.27(3.72)\end{array}$ & $\begin{array}{l}0.04(1.45) \\
0.36(1.65)\end{array}$ \\
\hline Satisfaction score & $\begin{array}{l}\text { Difference score mean (SE) } \\
\text { Percent change (SE) }\end{array}$ & $1-10$ & $\begin{array}{l}1.50(0.33) \\
43.61(10.22)\end{array}$ & $\begin{array}{l}-0.09(0.38) \\
12.45(11.94)\end{array}$ & $\begin{array}{l}-0.36(0.28) \\
-3.03(3.42)\end{array}$ \\
\hline Work related pain questions & $\begin{array}{l}\text { Difference score mean (SE) } \\
\text { Percent change (SE) }\end{array}$ & $1-10$ & $\begin{array}{l}-1.95(0.28) \\
-29.99(8.22)\end{array}$ & $\begin{array}{l}-0.48(0.34) \\
8.43(8.69)\end{array}$ & $\begin{array}{l}0.08(0.13) \\
2.34(4.13)\end{array}$ \\
\hline Work related disability questions & $\begin{array}{l}\text { Difference score mean (SE) } \\
\text { Percent change (SE) }\end{array}$ & $1-10$ & $\begin{array}{l}-1.52(0.35) \\
-39.6(5.06)\end{array}$ & $\begin{array}{l}-0.09(0.31) \\
-3.43(7.90)\end{array}$ & $\begin{array}{l}0.03(0.08) \\
12.8(8.55)\end{array}$ \\
\hline
\end{tabular}

Higher scores indicate greater function and increased satisfaction (SRQ), and less pain and less disability.

*Indicates significant change in mean scores between pretest and post-test $(p<0.01)$.

group. ${ }^{12}$ Brox et al reported significant improvements in subjects with shoulder impingement randomised to an exercise group compared to placebo at both 6 month and 2.5 year follow up periods. ${ }^{26} 27$ The odds ratio for success after arthroscopic decompression surgery compared to exercise was 1.5 , but results of the surgical intervention were not significantly different than results of the exercise programme after 2.5 years of follow up. Brox and colleagues' exercise intervention included supervised exercises with a physiotherapist twice a week, and home exercises on the other days. Exercises progressed from relaxed repetitive movements to strengthening over 3-6 months of treatment. ${ }^{26} 27$ Ginn et al report significant improvements in shoulder function in a physical therapy exercise treatment group compared to a control group in their randomised clinical trial of patients with shoulder pain believed to be of local mechanical origin. ${ }^{25}$ Treatments were individualised for each subject and included shoulder stretching and strengthening exercises, and "motor retraining aimed at restoring scapulohumeral rhythm" over a one month intervention period. ${ }^{25}$

Our results showed positive effects despite involving only five exercises completed independently by the workers at home after instruction, and 1-2 brief rechecks with the therapist. Our investigation is also unique in that the exercise programme was designed specifically based on identified motion and muscle activity alterations in construction workers routinely exposed to overhead work. ${ }^{10}{ }^{11}$ The magnitude of functional improvements can be evaluated relative to the standard error of the measurement (SEM) and smallest real difference (SRD) values for the SRQ. The SRD represents the smallest measurement change for an individual subject that can be interpreted as a real difference. ${ }^{28}$

Based on the previous analysis of psychometric properties of the SRQ, ${ }^{19}$ the SEM can be calculated $(\mathrm{SD} \times \sqrt{(\mathrm{l}-\mathrm{r}))}$ as 2.1 SRQ points and the SRD at $95 \%$ confidence $(S E M \times \sqrt{2} \times 1.96)$ as 5.8 SRQ points. ${ }^{28}$ The mean change in SRQ scores in the intervention group was therefore approximately twice the SRD. When considering individual subject's values, $67 \%$ of the intervention subjects showed positive improvements on the SRQ at post-test that were at or above the SRD threshold. SRQ improvements were further explored using the available data on compliance. Subjects categorised as having high compliance $(75 \%$ or greater frequency of completion of the exercise programme, $\mathrm{n}=9$ ) showed an average increase in SRQ score of 20.1, while subjects categorised as having low compliance (less than $25 \%$ frequency of completion, $\mathrm{n}=3$ ) showed an average SRQ change score of -1.6. The high compliance subjects were also those who showed the most consistent increases in repetitions (generally three sets of 20 at the completion of the programme) and greatest increases in resistance (average increase $22 \mathrm{~kg}$ ). Subjects with intermediate compliance levels showed more modest improvements in SRQ scores. This compliance data support the premise that it was the intervention, rather than other possible factors (attention of a therapist, placebo effect, etc) that resulted in reductions in pain and improvements in function for the intervention group. However, the compliance data should be interpreted cautiously since only $61 \%$ of the intervention subjects returned completed daily exercise logs, and the accuracy of these logs is also dependent on subject self report. Collection of accurate compliance data remains a challenge in clinical trials requiring substantive patient involvement in the intervention.

Although significant improvements were obtained, clearly intervention subjects did not obtain similar function and pain scores on average as the asymptomatic group (table 3 ), and an intervention of different intensity may be of greater benefit. Specific testing of "dose-response" relations for exercise programmes would help to address this issue. Also, improved compliance, the inclusion of therapeutic modalities, additional exercises, different selection of exercises, or medication in conjunction with the home exercise programme are all possible factors that might increase the benefit to the intervention group. In addition, despite the two scheduled rechecks, it is possible that intervention subjects may not have performed the exercises as instructed, resulting in an exercise programme that was less standardised than planned. This potential change in performance could have resulted in a less effective outcome. Further study is needed to help clarify all reasons for differential effects across intervention subjects. Because of the significant changes in function and reductions in pain showed by the intervention group, it is believed that the home exercise programme as intended was both efficacious and effective for this group. Optimally, long term follow up studies would allow determination of whether or not reduction of pain and improvements in function are of sufficient magnitude to result in reduced rates of rotator cuff surgeries and shoulder medical disability claims in these workers.

Given the lower initial functional levels as measured by SRQ scores at pretest in the intervention group, one might expect greater improvements over time in this group compared to the symptomatic control group. However, the SRQ change scores remained significantly higher in the intervention group compared to the control group, even after controlling for the pretest scores. Additionally, all other outcome variables, which did not differ between the intervention and control groups at pretest, showed significant improvements in the intervention group compared to the control group at post-test. Furthermore, significant differences remained for all variables except satisfaction scores when completing intention to treat analyses. Intention to treat analyses adjust for the possibility that subjects lost to follow up may have responded less favourably than other intervention subjects and more favourably than other control subjects. $^{23}$ 
As indicated earlier, two of the outcome measures used in this investigation were modified from the SPADI to create more specific work related pain and disability questions. The psychometric properties of these modified questions are not fully known. To explore this issue, we used a Pearson correlation value of the modified SPADI scores to the SRQ scores to assess construct validity. Additional work related pain questions showed a negative correlation of 0.80 and additional work related disability questions a negative correlation of 0.75 , with higher SRQ function associated with lower work related pain and disability. Also, the significant differences in these outcome measures noted in the intervention group compared to the control group at posttest suggest that these additional questions are adequately responsive to identify intervention effects. Further investigation is warranted to fully explore the usefulness of these additional work related questions as an adjunct to previously validated scales.

In the United States, many construction contractors are utilising standard group stretching and strengthening exercise programmes with workers prior to initiating daily work. These programmes generally attempt to provide a "warm up" for workers for a number of joints, and are not targeted specifically to motion or muscle activity alterations previously identified in workers. Improving the design of these programmes and testing them for positive preventive effects in reducing occupationally related musculoskeletal symptoms deserves further exploration. Similarly, although our exercises were targeted at specific shoulder muscles and the glenohumeral joint capsule, little scientific data is available comparing the physiological effectiveness of differing exercises intended to target the same anatomical structures. For example, we instructed subjects in a posterior capsule stretch commonly used in clinical practice and described in the literature. Yet other posterior shoulder stretches have been advocated $^{29}$ and it is unknown which might more directly stretch the joint capsule rather than the full posterior shoulder region, or if the source of posterior tightness derives from the capsule or musculature. Further research is needed to better understand the mechanics and mechanisms of shoulder impingement and help determine the most effective exercise programmes to reduce shoulder symptoms and improve function.

Interestingly, despite denying any history of symptoms and passing a clinical screening, the asymptomatic group did not show perfect scores on pain or function measures. Our impression after working with construction journeymen is that typically they are reluctant to use medication or medical services, and that they tend to minimise symptoms, with expectations that pain is just a part of the job, and that "everything hurts" to a certain extent. Our data indicate that asymptomatic subjects do not have scores showing maximum function and absence of pain or disability, which supports these impressions and speaks to the inherent physical stress of this type of work.

A number of limitations to the generalisability of this study should be noted. The volunteer convenience sample tested may impact the generalisability of results. Subjects included male construction workers only. One female volunteered but did not meet the clinical inclusion criteria. Workers in these trades are generally $98 \%$ male, and it is uncertain if response to exercises would be similar in female workers. Additionally, the majority of subjects in the study experienced chronic intermittent shoulder symptoms, with five years as the average time since initial onset of symptoms. More acute subjects may respond differently to a similar exercise programme. Further, subjects for this study were clinically screened to ensure that symptoms were consistent with shoulder impingement and rotator cuff tendonitis, and were instructed in the exercises by licensed physical therapists who were also available to recheck and answer questions from subjects. Similar results should not be expected in the treatment of non-specific shoulder pain, and persons undertaking an exercise programme are advised to consult a health care professional to ensure their appropriateness for an exercise intervention.

Because the intervention group received two rechecks, one in person and one generally by phone, this group received more attention of a therapist than the control group, and this differential attention may have impacted the results. However, this differential attention between groups was modest, as pretest and post-test time averaged 60-75 minutes each session, whereas recheck time was generally 1520 minutes each contact. The influence of therapist attention might be clarified in further studies by including an exercise group that receives no additional follow up visits, something we did not design into this analysis because this could result in subjects not properly performing the exercises or reducing compliance. Alternatively, in subsequent investigations the control groups could also receive rechecks or phone contacts. In a different investigation of the effectiveness of a home exercise programme for knee pain, subjects were randomly assigned to exercise therapy, monthly telephone contact, exercise therapy plus telephone contact, or no intervention. ${ }^{30}$ Regular telephone contact alone did not reduce pain in that investigation. ${ }^{30}$ The greater improvements seen for high compliance subjects in our investigation also support the premise that the positive outcomes are not likely to be due solely to attention of the therapist.

Optimally, a placebo exercise programme would allow for investigation of the magnitude of placebo effects in this type of study. However, due to the paucity of data regarding the effectiveness of exercise programmes for shoulder pain, a placebo exercise programme that was both plausible to the subjects to allow for blinding and yet known to not be efficacious was not identifiable by the authors. Subjects were blinded to their initial scores when completing the posttesting, and patients were asked to rate their current status rather than a change in status, so recall bias should not have substantially impacted the results. Further study assessing more objective measures of the physiological effects of the exercise intervention would be beneficial since physiological variables are less likely impacted by possible placebo effects.

A final potential confounding factor in this investigation was variation in the post-test time between subjects (8-12 weeks). Not surprisingly, it was not always possible for subjects to return for the post-test exactly at the completion of the eight week period. The majority of subjects discontinued the exercise programme after 8-9 weeks, regardless of the scheduled follow up time. Therefore, the apparent effectiveness of the exercises might appear to be less for subjects not seen for followup until the 12th week. Inspection of the data did not reveal any identifiable trends where the differences between groups could be related to longer time to follow up in one group or another.

\section{Conclusions}

A shoulder home exercise programme showed significant positive improvements in shoulder function and reductions in symptoms for construction workers with routine exposure to overhead work. Any potential long term value of exercise programmes in reducing rates of rotator cuff surgeries and shoulder medical disability claims in these workers deserves further investigation.

\section{APPENDIX A}

Copyright owned by The Journal of Bone and Joint Surgery, Inc. Full citation: L'Insalata JC, Warren RF, Cohen SB, et al. A 
self-administered questionnaire for assessment of symptoms and function of the shoulder. J Bone Joint Surg 1997;79-A(5): 738-48.

For brevity, where simultaneous questions use the same answer format, the formats are not repeated.

\section{Shoulder Rating Questionnaire}

Please answer the following questions regarding the shoulder for which you have been evaluated or treated. If a question does not apply to you, leave that question blank. If you indicated that both shoulders have been evaluated or treated, please complete a separate questionnaire for each shoulder and mark the corresponding side (right or left) at the top of each form.

1. Considering all the ways that your shoulder affects you, circle a number on the scale below for how well you are doing.

Very poorly $\{12345678910\}$ Very well

The following questions refer to pain.

2. During the past month, how would you describe the usual pain in your shoulder at rest?
(A) Very severe
(B) Severe
(C) Moderate
(D) Mild
(E) None

3. During the past month, how would you describe the usual pain in your shoulder during activities?

4. During the past month, how often did the pain in your shoulder make it difficult for you to sleep at night?
(A) Every day
(B) Several days per week
(C) One day per week
(D) Less than one day per week
(E) Never

5. During the past month, how often have you had severe pain in your shoulder?

The following questions refer to daily activities.

6. Considering all the ways you use your shoulder during daily personal and household activities (i.e. dressing, washing, driving, household chores, etc), how would you describe your ability to use your shoulder?
(A) Very severe limitation; unable
(B) Severe limitation
(C) Moderate limitation
(D) Mild limitation
(E) No limitation

Questions 7-11: During the past month, how much difficulty have you had in each of the following activities due to your shoulder?

7. Putting on or removing a pullover, sweater or shirt:
(A) Unable
(B) Severe difficulty
(C) Moderate difficulty
(D) Mild difficulty
(E) No difficulty

8. Combing or brushing your hair.

9. Reaching shelves that are above your head.
10. Scratching or washing your lower back with your hand.

11. Lifting or carrying a full bag of groceries (8-10 pounds).

The following questions refer to recreational or athletic activities.

12. Considering all the ways you use your shoulder during recreational or athletic activities (i.e. baseball, golf, aerobics, gardening, etc), how would you describe the function of your shoulder?

(A) Very severe limitation; unable

(B) Severe limitation

(C) Moderate limitation

(D) Mild limitation

(E) No limitation

13. During the past month, how much difficulty have you had throwing a ball overhand or serving in tennis due to your shoulder?

14. List one activity (recreational or athletic) that you particularly enjoy and then select the degree of limitation you have, if any, due to your shoulder.

Activity

The following questions refer to work.

15. During the past month, what has been your main form of work?

(A) Paid work (list type of work)

(B) Housework

(C) Schoolwork

(D) Unemployed

(E) Disabled due to your shoulder

(F) Disabled secondary to other causes

(G) Retired

If you answered D, E, F, or G to the above question, please skip questions 16-19 and go on to question 20.

16. During the past month, how often were you unable to do any of your usual work because of your shoulder?

(A) All days

(B) Several days per week

(C) One day per week

(D) Less than one day per week

(E) Never

17. During the past month, on the days that you did work, how often were you unable to do your work as carefully or as efficiently as you would like because of your shoulder?

18. During the past month, on the days that you did work, how often did you have to work a shorter day because of your shoulder?

19. During the past month, on the days that you did work, how often did you have to change the way that your usual work is done because of your shoulder?

The following questions refer to satisfaction and areas for improvement.

20. During the past month, how would you rate your overall degree of satisfaction with your shoulder?
(A) Poor
(B) Fair
(C) Good
(D) Very good
(E) Excellent 
21. Please rank the two areas in which you would most like to see improvement (place a 1 for the most important, a 2 for the second most important).

Pain ...............

Daily personal and household activities .............

Recreational or athletic activities

Work

\section{Authors' affiliations}

P M Ludewig, J D Borstad, Program in Physical Therapy, Department of Physical Medicine \& Rehabilitation, The University of Minnesota, Minneapolis, MN 55455, USA

This project was funded by the Center to Protect Worker's Rights, the Public Health Service, and the University of lowa, USA (grant \# U60/ CCU317202)

\section{REFERENCES}

1 National Institute for Occupational Safety and Health. Shoulder musculoskeletal disorders: evidence for work-relatedness. In:Bernard BP, ed. Musculoskeletal disorders and workplace factors-a critical review of epidemiologic evidence for work-related musculoskeletal disorders of the neck, upper extremity, and low back. 2nd ed. Publication no. 97-141: 1-72, Cincinnati, OH: US Department of Health and Human Services, Public Health Service, Centers for Disease Control and Prevention, 1997.

2 Bielle A, Hagberg M, Michaelson G. Occupational and individual factors in acute shoulder-neck disorders among industrial workers. Br J Ind Med $1981 ; 38: 356-63$.

3 Hagberg $M$, Wegman DH. Prevalence rates and odds ratios of shoulder-neck diseases in different occupational groups. Br J Ind Med 1987;44:602-10.

4 Cook TM, Rosecrance JC, Zimmermann CL. The University of lowa Construction Survey. Publication no. 010-96. Washington, DC: The Center to Protect Worker's Rights, 1996.

5 Fu FH, Harner CD, Klein AH. Shoulder impingement syndrome: a critical review. Clin Orthop Rel Res 1991;269:162-73.

6 Matsen F, Arntz CT. Subacromial impingement. In: Rockwood CA, Matsen F, eds. The shoulder. Philadelphia, PA: WB Saunders Co., 1990:623-46.

7 Zuckerman JD, Kummer FJ, Cuomo F, et al. The influence of coracoacromial arch anatomy on rotator cuff tears. J Shoulder Elbow Surg 1992;1:4-14.

8 Neer CS. Impingement lesions. Clin Orthop 1983;173:70-7.

9 Kamkar A, Irrgang JJ, Whitney SL. Nonoperative management of secondary shoulder impingement syndrome. J Orthop Sports Phys Ther 1993;17:212-24.

10 Ludewig PM, Cook TM. Alterations in shoulder kinematics and associated muscle activity in people with symptoms of shoulder impingement. Phys Ther 2000;80:276-91.
11 Ludewig PM, Cook TM. Translations of the humerus in persons with shoulder impingement symptoms. J Orthop Sports Phys Ther 2002;32:248-59.

12 Bang MD, Deyle GD. Comparison of supervised exercise with and without manual physical therapy for patients with shoulder impingement syndrome. J Orthop Sports Phys Ther 2000;30:126-37.

13 Wang $\mathrm{C}-\mathrm{H}, \mathrm{McCl}$ (ure $\mathrm{P}$, Pratt NE, et al. Stretching and strengthening exercises: their effect on three-dimensional scapular kinematics. Arch Phys Med Rehabil 1999;80:923-9.

14 Hawkins RJ, Abrams JS. Impingement syndrome in the absence of rotator cuff tear (stages 1 and 2). Orthop Clin North Am 1987;18:373-82.

15 LeRoux JL, Thomas E, Bonnel F, et al. Diagnostic value of clinical tests for shoulder impingement syndrome. Rev Rheum 1995;62:423-8.

16 Ure BM, Tiling R, Kirchner R, et al. The value of clinical shoulder examination in comparison with arthroscopy. A prospective study. Unfallchirurg 1993;96:382-6.

17 Kessel L, Watson M. The painful arc syndrome: clinical classification as a guide to management. J Bone Joint Surg 1977;59-B:166-72.

18 Calis $M$, Akgün K, Birtane M, et al. Diagnostic values of clinical diagnostic tests in subacromial impingement syndrome. Ann Rheum Dis 2000;59:44-7.

19 L'Insalata JC, Warren RF, Cohen SB, et al. A self-administered questionnaire for assessment of symptoms and function of the shoulder. J Bone Joint Surg 1997:79-A:738-48.

20 Williams JW, Holleman DRJ, Simel DL. Measuring shoulder function with the shoulder pain and disability index. J Rheumatol 1995;22:727-32.

21 Roach KE, Budiman-Mak E, Songsiridej N, et al. Development of a shoulder pain and disability index. Arthritis Care Res 1991;4:143-9.

22 Ludewig PM. Alterations in shoulder kinematics and associated muscle activity in persons with shoulder impingement syndrome. Doctoral dissertation. lowa City, IA: The University of lowa, 1998:50-72.

23 Miller ME, Morgan TM, Espeland MA, et al. Group comparisons involving missing data in clinical trials: a comparison of estimates and power (size) for some simple approaches. Statist Med $2001 ; 20: 2383-97$.

24 van der Windt DA, Koes BW, de Jong BA, et al. Shoulder disorders in general practice: incidence, patient characteristics, and management. Ann Rheum Dis 1995; 54:959-64.

25 Ginn KA, Herbert RD, Khouw W, et al. A randomized, controlled clinical trial of a treatment for shoulder pain. Phys Ther 1997;77:802-9.

26 Brox Jl, Gjengedal E, Uppheim G, et al. Arthroscopic surgery versus supervised exercises in patients with rotator cuff disease (stage II impingement syndrome): a prospective, randomized, controlled study in 125 patients with a 21/2-year follow-up. J Shoulder Elbow Surg 1999;8:102-11.

27 Brox Jl, Staff PH, Ljunggren AE, et al. Arthroscopic surgery compared with supervised exercises in patients with rotator cuff disease (stage II impingement syndrome). BMJ 1993;307:899-903.

28 Beckerman H, Roebroeck ME, Lankhorst GJ, et al. Smallest real difference, a link between reproducibility and responsiveness. Qual Life Res 2001; 10:571-8

29 Johansen RL, Callis M, Potts J, et al. A modified internal rotation stretching technique for overhand and throwing athletes. J Orthop Sports Phys Ther 1995;21:216-19.

30 Thomas KS, Muir KR, Doherty M, et al. Home based exercise programme for knee pain and knee osteoarthritis: randomised controlled trial. $B M J$ 2002;325:752-7. 